
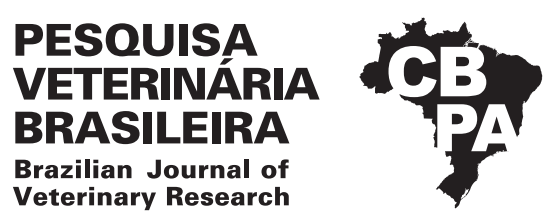

Pesq. Vet. Bras. 38(7):1278-1285, julho 2018 DOI: 10.1590/1678-5150-PVB-5289

Artigo Original

ISSN 0100-736X (Print)

ISSN 1678-5150 (Online)

\title{
Prevalência e susceptibilidade antimicrobiana de sorotipos de Salmonella spp. isolados de frangos vivos e carcaças no estado do Rio de Janeiro ${ }^{1}$
}

\author{
Daniela Q. Baptista ${ }^{2,3 *}$, André F.M. Santos ${ }^{2,4}$, Maria Helena C. Aquino², \\ Dayse L.C. Abreu², Dália P. Rodrigues ${ }^{4}$, Elmiro R. Nascimento ${ }^{2}$ e Virginia L.A. Pereira ${ }^{2}$
}

\begin{abstract}
Baptista D.Q., Santos A.F.M, Aquino M.H.C., Abreu D.L.C., Rodrigues D.P., Nascimento E.R. \& Pereira V.L.A. 2018. [Prevalence and antimicrobial susceptibility of Salmonella spp. serotypes in broiler chickens and carcasses in the State of Rio de Janeiro, Brazil] Prevalência e susceptiblidade antimicrobiana de sorotipos de Salmonella spp. isolados de frangos vivos e carcaças no estado do Rio de Janeiro. Pesquisa Veterinária Brasileira 38(7):1278-1285. Departamento de Saúde Coletiva Veterinária e Saúde Pública, Universidade Federal Fluminense, Rua Vital Brazil Filho 64, Niterói, RJ 24230-340, Brazil. E-mail: daniqb@yahoo.com

The presence of Salmonella spp. in poultry products and their by-products is a major challenge for commercial production. Data about the prevalence, the circulating serotypes and the antimicrobial susceptibility profile of Salmonella spp. strains in the State of Rio de Janeiro are scarce. Therefore, the aim of this study was to detect the presence of Salmonella spp. in live chickens and carcasses in slaughterhouses of the State of Rio de Janeiro, to identify the serotypes and to evaluate the antimicrobial susceptibility of these strains for fluoroquinolones and beta-lactams. Sixty cloacal swabs samples from broiler chickens and sixty samples of carcasses from six slaughterhouses under State Inspection were collected. The isolates were serotyped and resistance was tested to eight antimicrobials: enrofloxacin, ciprofloxacin, norfloxacin, cephalothin, ceftiofur, cefotaxime, amoxicillin/clavulanic acid and ampicillin by disc diffusion method. The results showed a prevalence of Salmonella spp. of $1.66 \%(1 / 60)$ in cloacal swabs samples and $26.66 \%(16 / 60)$ in carcasses. In cloacal swabs sample only Senftenberg (1.66\%) serotype was isolated. In total, seven different serotypes were obtained from carcasses: Senftenberg (15\%), followed by Mbandaka (8.3\%), Schwarzengrund (3.3\%), Cerro (3.3\%), Ohio (3.3\%), Minnesota (1.66\%) and Tennessee $(1.66 \%)$. Regarding antimicrobial susceptibility, $29(87.87 \%)$ isolates were sensitive to all antimicrobials tested and $4(12.12 \%)$ isolates were resistant to three or more beta-lactams antimicrobials. No susceptibility to fluoroquinolones was observed. These results showed a prevalence of Salmonella spp. higher than expected in slaughterhouses in the State of Rio de Janeiro, besides the presence of several serotypes of Salmonella spp. The resistance found for beta-lactams alerts to the spread of these strains through the food chain.
\end{abstract}

INDEX TERMS: Antimicrobial susceptibility, Salmonella spp., serotype, broilers, Rio de Janeiro, Brazil, poultry carcass, bacterioses.

\footnotetext{
${ }^{1}$ Recebido em 2 de julho de 2017.

Aceito para a publicação em 19 de julho de 2017.

2 Programa de Pós-Graduação em Higiene Veterinária e Tecnologia e Processamento Tecnológico de Produtos de Origem Animal, Faculdade de Medicina Veterinária, Universidade Federal Fluminense, Rua Vital Brasil 64, Santa Rosa, Niterói, RJ 24320-340, Brasil. *Autora para correspondência: daniqb@yahoo.com

${ }^{3}$ Coordenação Estadual de Defesa Sanitária Animal, Secretaria Estadual Agricultura e Pecuária do Estado do Rio de Janeiro, Alameda São Boaventura 770, Fonseca, Niterói, RJ 24120-191.

${ }^{4}$ Laboratório de Enterobactérias, Pavilhão Rocha Lima, Instituto Oswaldo Cruz/Fiocruz, Avenida Brasil 4365, Manguinhos, Rio de Janeiro, RJ 21040-360.
}

RESUMO.- A presença de Salmonella spp. em produtos de origem avícola e seus subprodutos se mostra um grande desafio para a produção comercial. Dados de prevalência, dos sorotipos circulantes e do perfil de susceptibilidade antimicrobiana de cepas de Salmonella spp. no Estado do Rio de Janeiro são escassos. Portanto, objetivou-se detectar a presença Salmonella spp. em frangos vivos e carcaças em matadouros do Estados do Rio de Janeiro, identificar os sorotipos e avaliar a susceptibilidade antimicrobiana dessas cepas para fluoroquinolonas e betalactâmicos. Foram coletadas 
60 amostras cloacais de frangos vivos e 60 amostras de carcaça de seis matadouros sob Inspeção Estadual (SIE). Os isolados foram sorotipificados e testados frente a oito antimicrobianos: enrofloxacina, ciprofloxacina, norfloxacina, cefalotina, ceftiofur, cefotaxima, amoxicilina/ácido clavulânico e ampicilina pelo método de difusão em disco. Os resultados mostraram uma prevalência de Salmonella spp. de 1,66\% (1/60) em amostras de suabe de cloaca e de $26,66 \%$ (16/60) em carcaças. Em amostras de suabe de cloaca, somente o sorotipo Senftenberg $(1,66 \%)$ foi isolado. No total, foram isolados sete sorotipos diferentes nas carcaças: Senftenberg (15\%) o mais frequente, seguido por Mbandaka (8,3\%), Schwarzengrund (3,3\%), Cerro (3,3\%), Ohio (3,3\%), Minnesota (1,66\%) e Tennessee $(1,66 \%)$. Em relação à susceptibilidade antimicrobiana, 29 (87,87\%) isolados foram sensíveis a todos os antimicrobianos testados e $4(12,12 \%)$ isolados foram resistentes a pelo menos três antimicrobianos betalactâmicos ou mais. Não foi observada resistência às fluoroquinolonas. Os resultados encontrados demonstram uma prevalência de Salmonella spp. acima da esperada em matadouros do Estado do Rio de Janeiro, além da presença de vários sorotipos de Salmonella spp. A resistência encontrada para betalactâmicos alerta para a disseminação dessas cepas pela cadeia alimentar.

TERMOS DE INDEXAÇÃO: Susceptiblidade antimicrobiana, sorotipo, Salmonella spp., frango, carcaças, Rio de Janeiro, bacterioses.

\section{INTRODUÇÃO}

A salmonelose é uma das principais doenças que afetam a população mundial através da ingestão de alimentos contaminados (WHO 2016a). Geralmente está associada ao consumo de produtos de origem animal, sendo que as aves e seus subprodutos têm sido incriminados como as fontes mais prováveis de contaminação (Jackson et al. 2013, Voss-Rech et al. 2015, WHO 2016a). Nos Estados Unidos, anualmente, são estimados mais de 1.000,000 de casos da doença, 19,000 hospitalizações e 380 óbitos em decorrência da salmonelose (CDC 2012). No Brasil, Salmonella spp. é apontada como o principal microrganismo envolvido nos surtos de doenças alimentares em geral, identificada em 7,5\% dos casos entre os anos de 2007 e 2016 (Brasil 2016b).

Em função do risco de infecção associado aos produtos de origem avícola e a prevalência da resistência antimicrobiana, o controle da Salmonella spp. se mostra um grande desafio para a produção comercial, além da diversidade de sorotipos existentes (Shah et al. 2017). Com o objetivo de resguardar a produção avícola e a saúde pública, o Ministério da Agricultura, Pecuária e Abastecimento (MAPA), implementou, em 1994, o Programa Nacional de Sanidade Avícola (PNSA) (Brasil 1994). Dentro desse programa, em 2003, iniciou-se o monitoramento de Salmonella spp. em carcaças de frango e perus em matadouros inspecionados pelo Sistema de Inspeção Federal (SIF) (Brasil 2016a).

Entretanto, são escassos os estudos sobre a prevalência de Salmonella spp. no Estado do Rio de Janeiro (Asensi et al. 2009,Medeiros et al. 2011, Panzenhagen et al. 2016), que na década de 60 foi o mais importante polo avícola do Brasil (UBABEF 2011). Atualmente, a avicultura do estado produz muito aquém do registrado para outros estados (ABPA 2016). Contudo, o Rio de Janeiro é o estado do país com o segundo maior valor de despesa média familiar com alimentação, atrás apenas de São Paulo (IBGE 2015). Logo, o conhecimento do estado sanitário do plantel fluminense e das condições higiênico-sanitárias dos produtos derivados se torna essencial.

Salmonella spp. podem ser disseminadas pela cadeia alimentar (WHO 2016a). Sua prevalência em carcaças é variável em diferentes locais do Brasil, oscilando entre 6,67\% e 58\% (Fuzihara et al. 2000, Oliveira et al. 2006, Moreira et al. 2008, Duarte et al. 2009, Cardoso et al. 2015, Panzenhagen et al. 2016, Yamatogi et al. 2016), assim como a diversidade de sorotipos isolados (Costa et al. 2013). Consequentemente, boas práticas de produção são de extrema importância, principalmente dentro dos matadouros (Yamatogi et al. 2016). A ocorrência de Salmonella spp. também é descrita na granja, com variações entre 1,95\% e 11,4\% (Boni et al. 2011, Ravagnani et al. 2012, Giombelli \& Gloria 2014, Voss-Rech et al. 2015, Pandini et al. 2015). Em relação aos frangos vivos, a frequência relatada é muito baixa, cerca de 0,2\% (Ravagnani et al. 2012, Mezalira et al. 2014, Bezerra et al. 2016).

O conhecimento dos sorotipos circulantes representa uma importante ferramenta epidemiológica em qualquer país. Fatores genéticos podem influenciar a disseminação de certos sorotipos contribuindo para uma mudança na população bacteriana circulante (Costa et al. 2013). Ademais, a sorotipificação das cepas provenientes da cadeia avícola é importante para estimar a contaminação cruzada e explorar as relações entre a bactéria, o meio ambiente e as carcaças (Yamatogi et al. 2016). Muitos sorotipos de Salmonella spp. estão relacionados a casos de salmonelose por consumo de outros alimentos e não por consumo de carne de frango, como por exemplo: frutas (Jackson et al. 2013), verduras (Gill et al. 2003, Jackson et al. 2013), cereais de bebê (Rushdy et al. 1998), pimenta preta (Van Doren et al. 2013) e manjericão (Zweifel \& Stephan 2012), ou pelo contato com aves vivas (CDC 2014).

Outro fator relevante em relação à Salmonella spp. é a sua resistência a antimicrobianos. 0 uso prolongado e a baixa dose destes medicamentos utilizados como promotores de crescimento proporcionam o ambiente ideal de pressão seletiva para o surgimento de cepas resistentes (Van Boeckel et al. 2015). A circulação dessas cepas na cadeia alimentar influencia negativamente na terapêutica em casos graves de salmonelose no ser humano, tornando o antimicrobiano ineficaz e podendo levar ao óbito. (WHO 2016b). As variações na resistência das cepas isoladas podem estar associadas com o país, com o ano, com o tipo de criação, com a granja de procedência do lote, com o sorotipo da Salmonella spp. e, particularmente, com o antimicrobiano testado. Em vários estudos não foi possível avaliar individualmente a influência desses fatores, porém algumas tendências podem ser observadas. Por exemplo, pode-se citar a diferença de resistência encontrada entre aves de postura e frangos de corte. Para evitar resíduos de antimicrobianos em ovos, as aves de postura receberiam menos antimicrobianos do que as aves de corte, dessa forma cepas resistentes seriam encontradas com menos frequência nesse tipo de produção (Gyles 2008).

As fluoroquinolonas e as cefalosporinas de terceira geração, que pertencem ao grupo dos betalactâmicos, são medicamentos considerados criticamente importantes por serem utilizados tanto na medicina humana quanto na medicina veterinária (OIE 2015, WHO 2016b); também são utilizados no tratamento de casos severos de salmonelose humana (WHO 2016b), o 
que ressalta a importância do monitoramento e controle da disseminação de cepas resistentes a estes medicamentos.

O objetivo desse trabalho foi detectar a presença de Salmonella spp. em frangos vivos e carcaças em matadouros do Estados do Rio de Janeiro, identificar os sorotipos e avaliar a susceptibilidade antimicrobiana dessas cepas para fluoroquinolonas e betalactâmicos.

\section{MATERIAL E MÉTODOS}

Amostras. As amostras analisadas foram provenientes de seis matadouros (A, B, C, D, E, F) sob a fiscalização do Serviço de Inspeção Estadual (SIE), sendo um localizado na Região Metropolitana, quatro na Região Serrana e um na Região Centro-Sul Fluminense do estado do Rio de Janeiro.

Em cada coleta, obteve-se 10 amostras de cloaca de 40 frangos vivos com auxilio de suabe, sendo cada amostra composta por pools de quatro aves, além de 10 carcaças, todas provenientes do mesmo lote e de propriedades localizadas dentro do estado. 0 estudo atendeu a legislação vigente em relação ao número mínimo de carcaças a serem coletadas em matadouros fiscalizados pelo Serviço de Inspeção Federal, considerando-se uma prevalência esperada de $20 \%$ de Salmonella spp. (Brasil 2016a). As amostras de frangos vivos foram obtidas para efeito comparativo, sendo 10 amostras de cada extrato. No total, foram analisadas 60 amostras de suabe de cloaca e 60 carcaças, coletadas entre março e setembro de 2016.

As amostras cloacais de frangos vivos foram coletadas antes da pendura e acondicionadas em tubos contendo meio Cary e Blair. As carcaças foram escolhidas aleatoriamente após o gotejamento e armazenadas em sacos estéreis. Todas as amostras foram acondicionadas sob-refrigeração em caixas isotérmicas, encaminhadas para análise no Laboratório de Sanidade Avícola da Faculdade de Veterinária da Universidade Federal Fluminense e processadas em até cinco horas após a coleta.

Isolamento e identificação de Salmonella spp. As amostras cloacais dos frangos vivos foram processadas de acordo com a Portaria no 126 de 03/11/1995, do Ministério da Agricultura, Pecuária e Abastecimento (Brasil 1995).

Foi utilizado o método de rinsagem modificado para a avaliação bacteriológica das carcaças (USDA 1998). Para o processamento, o excesso de líquido da carcaça foi drenado de forma asséptica. Na sequência, $400 \mathrm{ml}$ de solução salina peptonada 1\% (SSP) foram vertidas em cada saco contendo as carcaças. Os sacos foram lacrados e agitados por 60 segundos, para que a solução pudesse alcançar toda a superfície das carcaças. Em seguida, $10 \mathrm{ml}$ da solução da lavagem foram transferidos para um recipiente estéril e incubados a $36 \pm 1^{\circ} \mathrm{C}$ por 16 a 20 horas. Seguiram-se as etapas de enriquecimento seletivo, plaqueamento seletivo e análises bioquímicas de acordo com a legislação brasileira (Brasil 2003).

Os isolados foram sorotipificados de acordo com o esquema White-Kauffmann-Le Minor (Grimont \& Weill 2007, Issenhuth-Jeanjean et al. 2014) no Laboratório de Enterobactérias do Instituto Oswaldo Cruz, Rio de Janeiro, Brasil

Teste de susceptibilidade antimicrobiana. Para avaliação da susceptibilidade antimicrobiana, foi realizado o teste de difusão em disco (CLSI 2014). 0 inóculo foi preparado em caldo Muller-Hinton $\left(\mathrm{KASVI}^{\circledR}\right.$ ) de acordo com o padrão 0.5 da escala de Mc Farland e semeado em ágar Mueller-Hinton (KASVI ${ }^{\circledR}$ ). Foram testados antibióticos do grupo das fluoroquinolonas: Enrofloxacina $(5 \mu \mathrm{g})$, Ciprofloxacina $(5 \mu \mathrm{g})$, Norfloxacina $(10 \mu \mathrm{g})$ e do grupo dos betalactâmicos: Cefalotina $(30 \mu \mathrm{g})$, Ceftiofur $(10 \mu \mathrm{g})$, Cefotaxima (30 $\mu \mathrm{g})$, Amoxicilina/Ácido Clavulânico $(20 / 10 \mu \mathrm{g})$ e Ampicilina $(30 \mu \mathrm{g})$.
A escolha dos antimicrobianos foi baseada na sua importância tanto na medicina veterinária, pelo uso frequente, quanto na medicina humana, por serem opções de tratamento.

Após a aposição dos discos, as placas foram incubadas a $35^{\circ} \mathrm{C}$ por 18 a 24 horas. Em seguida, os halos de inibição foram medidos em milímetros e a susceptibilidade das cepas foi classificada como resistente, intermediária ou sensível. 0 diâmetro do halo de cada disco foi avaliado de acordo com as recomendações do manual CLSI (2013, 2014).

Análise estatística. 0 Teste Qui-quadrado por independência (Ayres et al. 2007) foi aplicado para avaliar se houve diferença significativa entre os sorotipos de salmonelas isolados das carcaças. O programa BioStat, versão 5.3 foi utilizado para o cálculo.

\section{RESULTADOS E DISCUSSÃO}

\section{Prevalência de Salmonella spp. em frangos vivos e carcaças}

A partir dos estabelecimentos A e D não foi detectada nenhuma amostra positiva. Quanto às amostras cloacais de frangos vivos, apenas no matadouro E foi possível obter um isolado de Salmonella spp. (Quadro 1), o que provavelmente não representa a prevalência real de Salmonella spp. nos plantéis avícolas do estado do Rio de Janeiro. Assim como a baixa frequência obtida nesse trabalho (1,66\%), em granjas no Ceará, foram coletados 1.000 suabes de cloaca, sendo apenas $0,2 \%$ positivos (Bezerra et al. 2016). Resultados similares foram encontrados por Mezalira et al. (2014) no noroeste do Paraná, que ao analisarem 50 suabes de cloaca não detectaram a presença de Salmonella spp.. Em outro estudo no Paraná, onde foram coletados suabes de cloaca de 1.000 aves e suabes de arrasto dos mesmos galpões, também não foram obtidas amostras positivas, o que foi atribuído à ausência de Salmonella spp no ambiente (Ravagnani et al. 2012). Entretanto, pesquisas que utilizaram suabes de arrasto para isolar Salmonella spp. em outras granjas obtiveram resultados positivos, com a prevalência variando entre 1,95\% e 11,4\% (Boni et al. 2011, Giombelli \& Gloria 2014, Voss-Rech et al. 2015, Pandini et al. 2015). 0 método de coleta com auxílio de suabes de cloaca pode ter influenciado na baixa positividade observada nas amostras de frangos vivos desse estudo e naqueles com resultados semelhantes. De acordo com Van Hoorebeke et al. (2009), o suabe de cloaca parece não ser o melhor método de isolamento, subestimando a prevalência da Salmonella spp. quando esta é baixa no plantel, que aliada à sua excreção intermitente pelas aves podem explicar os resultados obtidos nesse estudo. Portanto, as diferentes prevalências desse microrganismo observadas em diversas regiões do país podem resultar da infecção dos lotes, da excreção

Quadro 1. Prevalência de Salmonella spp. em frangos vivos e carcaças em seis matadouros do estado do Rio de Janeiro, 2016

\begin{tabular}{cccc}
\hline Matadouro & Região & $\begin{array}{c}\text { Frangos vivos } \\
\mathrm{n}(\%)\end{array}$ & $\begin{array}{c}\text { Carcaças } \\
\mathrm{n}(\%)\end{array}$ \\
\hline A & & 0 & 0 \\
B & Serrana & 0 & $1(10)$ \\
C & & 0 & $7(10)$ \\
D & Centro-Sul Fluminense & 0 & 0 \\
E & Serrana & $1(10)$ & $3(30)$ \\
F & Metropolitana & 0 & $5(50)$ \\
Total & & $1(1,7)$ & $16(26,7)$
\end{tabular}


pelas fezes e das diferentes condições higiênico-sanitárias dos matadouros.

Em relação à análise das carcaças, amostras positivas para Salmonella spp. foram encontradas em quatro (B, C, E, F) dos seis matadouros analisados, o que correspondeu a $67 \%$ dos estabelecimentos visitados. No total, foram obtidos 33 isolados de Salmonella spp. de 16 das 60 carcaças analisadas (Quadro 1). 0 plano de monitoramento de Salmonella spp. em carcaças de frangos e perus do MAPA, preconiza uma prevalência esperada de $20 \%$ por ciclo amostral em SIF (Brasil 2016a). Analisando separadamente cada matadouro visitado em nosso estudo, nota-se que em três dos seis estabelecimentos, C, E e F, foi observado um limite acima do estabelecido pela legislação, com prevalência de $70 \%, 30 \%$ e $50 \%$, respectivamente, sendo dois localizados na Região Serrana e um na Metropolitana. Apenas no matadouro B, localizado na Região Serrana, foi observada uma prevalência dentro da preconizada pelo MAPA, com uma carcaça (10\%) positiva (Quadro 1). Apesar de não existir um plano estadual para esses estabelecimentos, espera-se que matadouros fiscalizados pelo SIE tenham um controle de qualidade similar aos fiscalizados pelo SIF.

A prevalência de $26,66 \%$ de Salmonella spp. em carcaças (Quadro 1) foi elevada quando comparada a estudo semelhante com sessenta carcaças de seis matadouros do estado do Rio de Janeiro, no qual foi observada uma positividade de carcaça de 6,67\% (Panzenhagen et al. 2016). Entretanto, variações de prevalência são descritas em diversos locais do Brasil. Cardoso et al. (2015) e Yamatogi et al. (2016), no estado de São Paulo, analisaram carcaças provenientes de matadouros com SIF e encontraram, respectivamente, $14,6 \%$ e $58 \%$ de carcaças positivas para Salmonella spp.. Em outra pesquisa realizada no mesmo estado, em 60 pequenos matadouros com condições precárias, a prevalência de Salmonella spp. foi de $42 \%$ nas carcaças analisadas (Fuzihara et al. 2000). Outros trabalhos no Ceará (Oliveira et al. 2006), em Pernambuco (Duarte et al. 2009) e em Goiás (Moreira et al. 2008), relatam uma prevalência de Salmonella spp. mais baixa do que a encontrada nesse estudo, $14,3 \%, 9,6 \%$ e $14,32 \%$, respectivamente. Sabe-se que boas práticas de fabricação e a aplicação do sistema APPCC podem influenciar a carga microbiana presente na linha de abate. 0 resultado desse estudo, onde foi observada a maior prevalência em carcaças, pode ter sido influenciado pela contaminação cruzada ocorrida no processamento tecnológico resultante do abate de lotes distintos no mesmo matadouro.

Giombelli \& Gloria (2014) não conseguiram correlacionar à ocorrência da Salmonella spp. na granja e em carcaças coletadas no matadouro. Em nosso estudo, podemos inferir que o único isolado de amostras de cloaca de frangos vivos pode estar relacionado com um isolado de carcaça do mesmo matadouro, pois os dois isolados pertencem ao mesmo sorotipo, compartilham do mesmo perfil de susceptibilidade antimicrobiana e foram isolados do mesmo lote, sugerindo fonte comum de contaminação (Quadro 2). Segundo Yamatogi et al. (2016), as fases que precedem o abate, como a captura e o transporte, estressam as aves. Esse estresse causa um distúrbio das funções intestinais, diminuindo a resistência da ave e aumentando a disseminação da bactéria pelas fezes (Marin \& Lainez 2009), o que pode elevar a contaminação da carcaça e a disseminação do patógeno na linha de produção (Yamatogi et al. 2016).

\section{Sorotipos prevalentes de Salmonella spp.}

Paralelamente, o estudo dos sorotipos de Salmonella spp. isolados se destaca como uma importante ferramenta epidemiológica, pois permite que os pesquisadores avaliem o aparecimento de sorotipos emergentes na cadeia de produção, além dos historicamente associados a carne de aves, como Enteritidis, Typhimurium e Heidelberg (Shah et al. 2017), estimando seus impactos na saúde humana (Yamatogi et al. 2016).

No presente trabalho, sete sorotipos de Salmonella spp. foram identificados em carcaças, sendo Senftenberg (15\%) o mais frequente e também o único isolado das amostras cloacais de frangos vivos (Quadro 3), seguido por Mbandaka $(8,3 \%)$, Schwarzengrund $(3,3 \%)$, Cerro $(3,3 \%)$, Ohio $(3,3 \%)$, Minnesota (1,66\%) e Tennessee(1,66\%). Em um isolado de carcaça não foi possível completar a caracterização antigênica $(1,66 \%)$ e em quatro carcaças foram isolados mais de um sorotipo de Salmonella spp. (Quadro 4). Apesar da diferença significativa entre a frequência de sorotipos circulantes $(p<0,05)$, esta diversidade era esperada, pois a variedade de sorotipos de Salmonella spp. em isolados de carcaças é grande, conforme descrito por inúmeros autores (Oliveira et al. 2006, Moreira et al. 2008, Duarte et al. 2009, Yamatogi et al. 2016). Entende-se que o monitoramento dos sorotipos circulantes na cadeia avícola do estado é importante para o conhecimento do papel dessas salmonelas em casos de surtos de salmonelose em humanos pela ingestão de produtos avícolas.

No estado do Rio de Janeiro são escassos os dados sobre os sorotipos circulantes. Panzenhagen et al. (2016) isolaram Salmonella Typhimurium e Salmonella Albany de carcaças resfriadas, enquanto Asensi et al. (2009) isolaram, predominantemente, Salmonella Enteritidis seguida de

Quadro 2. Perfil de susceptibilidade antimicrobiana de sorotipos de Salmonella spp. isolados de frango vivo e carcaças de matadouros no estado do Rio de Janeiro, 2016

\begin{tabular}{|c|c|c|c|c|c|c|c|c|c|c|}
\hline \multirow{3}{*}{ Sorotipo } & \multirow{3}{*}{ Matadouro } & \multirow{3}{*}{ Amostra } & \multicolumn{8}{|c|}{ Antimicrobianos } \\
\hline & & & \multicolumn{3}{|c|}{ Fluoroquinolonas } & \multicolumn{5}{|c|}{ Betalactâmicos } \\
\hline & & & ENO & NOR & CIP & AMC & CTF & CFL & CTX & AMP \\
\hline Senftenberg & B & Carcaça & I & $S$ & S & $\mathrm{R}$ & $\mathrm{R}$ & $\mathrm{R}$ & $\mathrm{R}$ & $\mathrm{R}$ \\
\hline Minnesota & E & Carcaça & $\mathrm{S}$ & $\mathrm{S}$ & $\mathrm{S}$ & $\mathrm{S}$ & $\mathrm{S}$ & $\mathrm{R}$ & $\mathrm{R}$ & $\mathrm{R}$ \\
\hline Senftenberg & $\mathrm{E}$ & Carcaça & $\mathrm{S}$ & $\mathrm{S}$ & $\mathrm{S}$ & $\mathrm{R}$ & I & $\mathrm{R}$ & $\mathrm{R}$ & $\mathrm{R}$ \\
\hline Senftenberg & E & Frango vivo & S & S & S & $\mathrm{R}$ & I & $\mathrm{R}$ & $\mathrm{R}$ & $\mathrm{R}$ \\
\hline
\end{tabular}

$\overline{\mathrm{ENO}}=$ enrofloxacina, $\mathrm{NOR}=$ norfloxacina, $\mathrm{CIP}=$ ciprofloxacina, $\mathrm{AMC}=$ amoxicilina com ácido clavulânico, $\mathrm{CTF}=$ ceftiofur, $\mathrm{CFL}=\mathrm{cefalotina}, \mathrm{CTX}=$ cefotaxima, $\mathrm{AMP}=$ ampicilina $\mathrm{S}$ = sensível, $\mathrm{R}$ = resistente, $\mathrm{I}$ = intermediário. 
Quadro 3. Sorotipos presentes de Salmonella spp. em matadouros no estado do Rio de Janeiro, 2016

\begin{tabular}{lcc}
\hline \multicolumn{1}{c}{ Sorotipo } & Carcaças n (\%) & Frangos vivos n (\%) \\
\hline Senftenberg & $9(15)$ & $1(1,7)$ \\
Mbandaka & $5(8,3)$ & 0 \\
Schwarzengrund & $2(3,3)$ & 0 \\
Cerro & $2(3,3)$ & 0 \\
Ohio & $2(3,3)$ & 0 \\
Minnesota & $1(1,7)$ & 0 \\
Tennessee & $1(1,7)$ & 0 \\
Salmonella enterica subsp. & $1(1,7)$ & 0
\end{tabular}

enterica (rugosa)

*Teste do Qui-quadrado por independência, p<0,05.

Quadro 4. Distribuição dos sorotipos de Salmonella spp. por matadouro e por carcaça analisada

\begin{tabular}{ccc}
\hline Matadouro & Carcaça & Sorotipo \\
\hline B & B-C11 & Senftenberg \\
C & C-C2 & Senftenberg \\
& C-C3 & Salmonella enterica subsp. enterica (rugosa) \\
& C-C4 & Senftenberg \\
& C-C6 & Senftenberg \\
& C-C7 & Senftenberg, \\
& C-C8 & Senftenberg \\
& C-C9 & Senftenberg \\
E & E-C2 & Schwarzengrund \\
& E-C4 & Senftenberg \\
& E-C8 & Minnesota \\
F & F-C2 & Schwarzengrund \\
& F-C5 & Mbandaka \\
& F-C6 & Mbandaka, Cerro, Ohio, \\
& & Salmonella enterica subsp. enterica $(0: 6,7)$ \\
& F-C7 & Mbandaka, Cerro, Ohio \\
& F-C10 & Mbandaka, Tennessee, Senftenberg
\end{tabular}

Salmonella Mbandaka, Salmonella Saintpaul e Salmonella enterica subsp. houtenae em carcaças congeladas adquiridas na cidade do Rio de Janeiro. Os dois trabalhos, ao contrário dessa pesquisa, encontraram os dois sorotipos de Salmonella spp. mais incriminados em casos de salmonelose humana por ingestão de produtos avícolas, ou seja, Salmonella Enteritidis e Salmonella Typhimurium. 0 monitoramento desses dois sorotipos de Salmonella na cadeia de produção avícola brasileira tem sido cada vez mais intenso o que pode ter influenciado na ausência dos mesmos nas amostras analisadas nesse estudo.

Destaca-se nesse estudo, o sorotipo Senftenberg, por ser ter sido o mais prevalente, sendo muito isolado atualmente no Brasil, tanto em produtos avícolas (Costa et al. 2013, Mattiello et al. 2015, Yamatogi et al. 2016) como em amostras provenientes de granjas (Boni et al. 2011, Mattiello et al. 2015). Apesar de estar listado como um dos sorotipos mais comumente isolados em produtos avícolas nos Estados Unidos, não foi encontrada relação significativa do consumo desses alimentos com casos de salmonelose humana ocasionada por Salmonella Senftenberg naquele país (Shah et al. 2017). Entretanto, surtos por esse sorotipo foram associados à ingestão de outros tipos de alimentos, como carne bovina, frutas e verduras (Jackson et al. 2013), cereais de bebê (Rushdy et al. 1998), manjericão (Zweifel \& Stephan 2012) e pimenta preta (Van Doren et al. 2013). Shirota et al. (2012) relataram que Salmonella Senftenberg é um patógeno persistente, resiste a altas temperaturas, contaminando primeiro a ração, espalhando-se pelo ambiente e, posteriormente para as aves, sendo prudente o seu monitoramento na alimentação animal e na contaminação de produtos avícolas. Todavia, sua epidemiologia, ecologia e virulência são pouco conhecidas, necessitando de mais estudos (Shah et al. 2017).

O sorotipo Mbandaka, encontrado em cinco carcaças analisadas, foi recentemente associado a um surto de salmonelose em humanos após o contato com galinhas de fundo de quintal nos Estados Unidos (CDC 2014). Na cadeia avícola brasileira, Salmonella Mbandaka é isolada com frequência (Fuzihara et al. 2000, Asensi et al. 2009, Duarte et al. 2009, Costa et al. 2013, Voss-Rech et al. 2015, Pandini et al. 2015, Yamatogi et al. 2016). Porém, assim como o sorotipo Senftenberg, não tem sido associado à ocorrência de salmonelose humana por consumo de carne de frango (Jackson et al. 2013, Shah et al. 2017). Alguns casos de salmonelose por este sorotipo foram relacionados ao consumo de carne de porco e ao consumo de brotos (Jackson et al. 2013), como por exemplo, o de alfafa (Gill et al. 2003).

Nesse estudo, outros sorotipos encontrados em menor proporção foram Schwarzengrund, Cerro, Ohio, Minnesota e Tennessee. De acordo com Shah et al. (2017), surtos de salmonelose no homem ligados ao sorotipo Schwarzengrund foram relacionados à ingestão de carne de frango comprada em mercado de aves vivas nos Estados Unidos.

Entre 2007 e 2011 houve um aumento significativo no isolamento de Salmonella Senftenberg e Salmonella Mbandaka em carcaças e subprodutos avícolas, sugerindo que são sorotipos emergentes. Sorotipos como Schwarzengrund, Minnesota e Tennessee também foram isolados com frequência nesses produtos. 0 aumento desses sorotipos pode estar relacionado ao nicho deixado pela redução na ocorrência de Salmonella Enteritidis. Essa diminuição é, provavelmente, um reflexo das ações implementadas pelo PNSA na tentativa de controle desse sorotipo e do sorotipo Typhimurium em granjas de reprodução (Costa et al. 2013). Tais dados corroboram com os resultados encontrados nessa pesquisa.

Diversos sorotipos de Salmonella podem ser encontrados nas aves e nos seus subprodutos. Entretanto, se faz necessário um estudo mais aprofundado do potencial patogênico de cada sorotipo em causar doença em humanos e o monitoramento e compilação de dados relacionados à prevalência desses sorotipos na salmonelose humana no Brasil.

\section{Perfil de susceptibilidade antimicrobiana}

Dos trinta e três isolados avaliados, vinte e nove $(87,87 \%)$ foram sensíveis a todos os antimicrobianos testados e somente quatro $(12,12 \%)$ se mostraram resistentes a pelo menos um antimicrobiano (Quadro 2). Contudo, esse percentual de resistência é baixo quando comparado a outros trabalhos que relatam um percentual entre $39 \%$ e $100 \%$ de resistência a pelo menos um dos antimicrobianos testados em cepas de Salmonella spp. no Brasil (Oliveira et al. 2006, Duarte et al. 2009, Costa et al. 2013, Fitch et al. 2016, Mattiello et al. 2015, Pandini et al. 2015, Bezerra et al. 2016, Yamatogi et al. 2016). 
Em relação às fluoroquinolonas, todos os isolados foram sensíveis a norfloxacina e a ciprofloxacina. Panzenhagen et al. (2016), em estudo no estado do Rio de Janeiro, relataram que as cepas de Salmonella spp. isoladas foram sensíveis as fluoroquinolonas testadas. Esses resultados sugerem que a resistência as fluoroquinolonas não é frequente em cepas circulantes no estado do Rio de Janeiro. Como sugerido por Gyles (2008), pode-se notar uma tendência no padrão de resistência em função da região onde a cepa foi isolada. Entretanto são necessários mais estudos sobre a resistência das cepas de Salmonella spp. circulantes na cadeia avícola do estado.

Costa et al. (2013), Fitch et al. (2016), Voss-Rech et al. (2015) e Yamatogi et al. (2016) também relatam alta susceptibilidade às fluoroquinolonas em estudos realizados no Brasil. Porém, um percentual de cepas, variando entre $4.9 \%$ e $19.2 \%$, que apresentaram resistência à enrofloxacina foi observado em estudos realizados no Brasil (Fitch et al. 2016, Medeiros et al. 2011, Yamatogi et al. 2016). Nesse trabalho, apenas um isolado demonstrou susceptibilidade intermediária à enrofloxacina (Quadro 2). Observa-se que este antimicrobiano é usado exclusivamente na medicina veterinária e seu uso indiscriminado nas criações avícolas pode resultar em aumento da resistência, como já relatado em estudos em outros países (Antunes et al. 2003, Donado-Godoy et al. 2012). No entanto, de acordo com a maioria dos autores (Gyles 2008, Duarte et al. 2009, Costa et al. 2013, Fitch et al. 2016, Yamatogi et al. 2016), tem se observado baixa resistência às fluoroquinolonas no Brasil, principalmente à ciprofloxacina, importante antimicrobiano utilizado no tratamento de casos graves de salmonelose no ser humano (WHO 2016a).

A resistência a betalactâmicos foi observada em quatro amostras. Todas as quatro cepas foram resistentes à ampicilina, a cefalotina e a cefotaxima, três à amoxicilina/ácido clavulânico, uma ao ceftiofur e duas apresentaram resistência intermediária também ao ceftiofur (Quadro 2). Em uma análise de 1.939 amostras de Salmonella spp. provenientes do Programa de Monitoramento desse patógeno em estabelecimentos com SIF (Brasil 2016a), 62\% dos isolados foram resistentes ao menos um dos antimicrobianos testados. 0 maior índice de resistência foi aos betalactâmicos (21,4\%), sendo $10 \%$ das amostras resistentes à ampicilina (Fitch et al. 2016). Esses resultados condizem com os encontrados nesse trabalho, onde $12,12 \%$ das amostras testadas foram resistentes a este antimicrobiano, além de outros betalactâmicos, como a cefalotina e a cefotaxima. Além disso, Medeiros et al. (2011) também encontraram cepas com um alto índice de resistência aos betalactâmicos, principalmente à ampicilina e ao ceftiofur. A resistência observada aos betalactâmicos nessa pesquisa é preocupante, pois foi observada resistência à cefalosporinas de terceira geração, como ao ceftiofur, antimicrobiano geralmente administrado intraovo ou no primeiro dia de vida do pintinho, visando inibir a contaminação por Salmonella spp. provenientes das matrizes (Palermo-Neto \& Borsoi 2013).

A transmissão horizontal de genes por plasmídios que codificam as betalactamases são uma constante preocupação no controle da resistência aos betalactâmicos (Gyles 2008). As cefalosporinas de terceira geração assim como as quinolonas são consideradas medicamentos críticos no tratamento de salmonelose invasiva no homem (WHO 2016b), sendo a circulação de cepas resistentes a estes medicamentos um ponto importante de controle na cadeia alimentar, pois constituem uma ameaça à saúde pública.

Das quatro cepas resistentes, três pertenciam ao sorotipo Senftenberg e um ao sorotipo Minnesota. Segundo Shah et al. (2017), cepas multirresistentes de Salmonella Senftenberg, principalmente às cefalosporinas de terceira geração, foram isoladas de fontes animais, incluindo aves. Todavia, há relatos de cepas sensíveis a antimicrobianos que foram correlacionadas com casos de salmonelose em humanos em hospitais nos Estados Unidos (Kay et al. 2007), reiterando a importância do monitoramento dos sorotipos circulantes, além do estudo da resistência.

Uma pesquisa realizada no Brasil entre 2007 e 2011, com 12.582 cepas de Salmonella spp., aponta para o crescimento exponencial de cepas com perfis multirresistentes, incluindo as cefalosporinas de terceira geração, assim como o aumento do número de sorotipos com esse perfil, abrangendo os de alta e baixa prevalência, sugerindo a propagação destes no ambiente (Costa et al. 2013). Para evitar a propagação de cepas resistentes, é necessário que haja cautela e responsabilidade no uso de antimicrobianos na medicina veterinária e na medicina humana, além de um monitoramento epidemiológico contínuo.

\section{CONCLUSÕES}

A presença de Salmonella spp. foi detectada na maior parte dos matadouros e diversos sorotipos foram identificados, sendo o sorotipo Senftenberg o mais prevalente.

Em 50\% dos matadouros investigados foi observada uma prevalência acima da preconizada pela legislação de monitoramento do MAPA.

A resistência observada ao menos a três antimicrobianos betalactâmicos alerta para a disseminação dessas cepas pela cadeia alimentar, sendo uma ameaça à saúde pública, pois coloca em risco o tratamento de quadros clínicos graves de salmonelose em seres humanos.

Um estudo mais aprofundado do potencial patogênico dos sorotipos isolados de aves e de seus perfis de susceptibilidade antimicrobiana se faz necessário.

Agradecimentos.- À Dra. Dália dos Prazeres Rodrigues, do Laboratório de Enterobactérias da Fiocruz, pela sorotipificação dos isolados de Salmonella spp.

\section{REFERÊNCIAS}

ABPA 2016. Relatório Anual ABPA. 136p. Disponível em <http://abpa-br com.br/setores/avicultura/publicacoes/ relatorios-anuais > Acesso em 10 jan. 2017.

Asensi G.F., Reis E.M.F., Del Aguila E.M., Rodrigues D.P., Silva J.T. \& Paschoalin V.M.F. 2009. Detection of Escherichia coli and Salmonella in chicken rinse carcasses. Brit. Food J. 111(6):517-527. <http://dx.doi. org/10.1108/00070700910965989>

Antunes P., Réu C., Sousa J.C., Peixe L. \& Pestana N. 2003. Incidence of Salmonella from poultry products and their susceptibility to antimicrobial agents. Int. J. Food Microbiol. 82(2):97-103.<http://dx.doi.org/10.1016/ S0168-1605(02)00251-9><PMid:12568749>

Ayres M., Ayres-Júnior M., Ayres D.L. \& Santos A.A.S. 2007. BioEstat 5.3: aplicações estatísticas nas áreas das ciências biomédicas. Sociedade Civil Mamirauá, Tefé. 364p.

Bezerra W.G.A., Silva I.N.G., Vasconcelos R.H., Machado D.N., Lopes E.S., Lima S.V.G., Teixeira R.S.C., Lima J.B., Oliveira F.R. \& Maciel W.C. 2016. Isolation and antimicrobial resistance of Escherichia coli and Salmonella enterica subsp. enterica $(0: 6,8)$ in broiler chickens. Acta Scient. Vet. 44:1-7. 
Boni H.F.K., Carrijo A.S. \& Fascina V.B. 2011. Ocorrência de "Salmonella" spp. em aviários e abatedouro de frangos de corte na região central de Mato Grosso do Sul. Revta Bras. Saúde. Prod. Anim. 12(1):84-95.

Brasil 1994. Portaria Ministerial no193, de 19 de setembro de 1994, institui o Programa Nacional de Sanidade Avícola no âmbito do DAS e cria o Comitê Consultivo do Programa de Sanidade Avícola. Ministério da Agricultura, Pecuária e do Abastecimento, Diário Oficial da União, Seção 1.

Brasil 1995. Normas de credenciamento e monitoramento de laboratórios de diagnóstico das salmoneloses aviárias ( $S$. Enteritidis, $S$. Gallinarum, $S$. Pullorum e $S$. Typhimurium). Portaria no126, de 3 de novembro de 1995, Ministério da Agricultura, Pecuária e Abastecimento, Diário Oficial da União, Seção 1.

Brasil 2003. Oficializar os métodos analíticos oficiais para análises microbiológicas para controle de produtos de origem animal e água. Instrução Normativa nº62, de 26 de agosto de 2003, Secretaria da Defesa Agropecuária (SDA), Ministério da Agricultura, Pecuária e do Abastecimento, Diário Oficial da União, Seção 1.

Brasil 2016a. Instrução Normativa no 20, de 21 de outubro de 2016, estabelece o Controle e o Monitoramento de Salmonella spp. nos Estabelecimentos Avícolas Comerciais de Frangos e Perus de Corte e nos Estabelecimentos de Abate de Frangos, Galinhas, Perus de Corte e Reprodução, registrados no Serviço de Inspeção Federal (SIF). Secretaria da Defesa Agropecuária (SDA), Ministério da Agricultura, Pecuária e do Abastecimento. Diário Oficial da União, Seção 1.

Brasil 2016b. Surtos de doenças transmitidas por alimentos no Brasil. Ministério da Saúde. Disponível em <http://u.saude.gov.br/images/ pdf/2016/junho/08/Apresenta----o-Surtos-DTA -2016.pdf> Acesso em 2 jan. 2017.

Cardoso A., Kanashiro A., Stoppa G., Castro A., Luciano R. \& Tessari E. 2015. Ocorrência de Salmonella spp. em carcaças de frango provenientes de abatedouros do estado de São Paulo, Brasil, no período de 2000 a 2010. Revta Cient. Eletrôn. Med. Vet. 24:1-12.

CDC 2012. Pathogens causing US foodborne illnesses, hospitalizations, and deaths, 2000-2008. Disponível em <https://www.cdc.gov/salmonella/ index.html> Acesso em 10 nov. 2016.

CDC 2014. Multistate outbreak of human Salmonella infections linked to live poultry from a mail-order hatchery in Ohio: march-september 2013. Morbidity and Mortality Weekly Report 63(10):222. Disponível em <https:// www.cdc.gov/mmwr/pdf/wk/mm6310.pdf> Acesso em 10 nov. 2016.

CLSI 2013. Performance standards for antimicrobial disk and dilution susceptibility tests for bacteria isolated from animals: approved standard and supplement documents VET 01-A4 and VET01-S2. 4th ed. Clinical and Laboratory Standards Institute, Wayne, PA. 99p.

CLSI 2014. Perfomance standards for antimicrobial susceptibility testing: twenty-fourth informational supplement. Clinical and Laboratory Standards Institute, Wayne, PA. 230p.

Costa R.G., Festivo M.L., Araujo M.S., Reis E.M., Lázaro N.S. \& Rodrigues D.P. 2013. Antimicrobial susceptibility and serovars of Salmonella circulating in commercial poultry carcasses and poultry products in Brazil. J. Food Protect. 76(12):2011-2017. <http://dx.doi.org/10.4315/0362-028X. JFP-13-164><PMid:24290674>

Donado-Godoy P., Gardner I., Byrne B.A., Leon M., Perez-Gutierrez E., Ovalle M.V., Tafur M.A. \& Miller W. 2012. Prevalence, risk factors, and antimicrobial resistance profiles of Salmonella from commercial broiler farms in two important poultry-producing regions of Colombia. J. Food Protect. 75(5):874-883. <http://dx.doi.org/10.4315/0362-028X.JFP-11458> <PMid:22564936>

Duarte D.A.M., Ribeiro A.R., Vasconcelos A.M.M., Santos S.B., Silva J.V.D., Andrade P.L.A. \& Falcão L.S.P.C.A. 2009. Occurrence of Salmonellaspp.in broiler chicken carcasses and their susceptibility to antimicrobial agents. Braz. J. Microbiol. 40(3):569-573. <http://dx.doi.org/10.1590/S151783822009000300020><PMid:24031401>
Fitch F.M., Carmo-Rodrigues M.S., Oliveira V.G.S., Gaspari M.V., Santos A., Freitas J.B. \& Pignatari A.C.C. 2016. $\beta$-Lactam resistance genes: characterization, epidemiology, and first detection of bla CTX-M-1 and bla CTX-M-14 in Salmonella spp. isolated from poultry in Brazil, Ministry of Agriculture's Pathogen Reduction Program. Microbiol. Drug Resist. 22(2):164-171. <http://dx.doi.org/10.1089/mdr.2015.0143> <PMid:26380894>

Fuzihara T.O., Fernandes S.A. \& Franco B.D.G.M. 2000. Prevalence and dissemination of Salmonella serotypes along the slaughtering process in Brazilian small poultry slaughterhouses. J. Food Protect. 63(12):1749-1753. <http://dx.doi.org/10.4315/0362-028X-63.12.1749><PMid:11131902>

Gill C.J., Keene W.E., Mohle-Boetani J.C., Farrar J.A., Waller P.L., Hahn C.G. \& Cieslak P.R. 2003. Alfalfa seed decontamination in Salmonella outbreak. Emerg. Infect. Dis. 9(4):474-479.<http://dx.doi.org/10.3201/eid0904.020519> <PMid:12702229>

Giombelli A. \& Gloria M.B.A. 2014. Prevalence of Salmonella and Campylobacter on broiler chickens from farm to slaughter and efficiency of methods to remove visible fecal contamination. J. Food Protect. 77(11):1851-1859. <http://dx.doi.org/10.4315/0362-028X.JFP-14-200><PMid:25364917>

Grimont P.A.D. \& Weill F.X. 2007. Antigenic formulae of Salmonella serovars. 9th ed. WHO, Collaborating Centre for Reference and Research on Salmonella, Institut Pasteur, Paris.

Gyles C.L. 2008. Antimicrobial resistance in selected bacteria from poultry. Anim. Health Res. Rev. 9(2):149-158. <http://dx.doi.org/10.1017/ S1466252308001552><PMid:19102788>

IBGE 2015. Pesquisa de orçamentos familiares 2008-2009: despesas e rendimentos. Disponível em <http://www.ibge.gov.br/estadosat/perfil. php?sigla=rj> Acesso em 23 nov. 2015.

Issenhuth-Jeanjean S., Roggetin P., Mikoleit M., Guibourdnche M., Pinna E., Nair S., Fields P.I. \& Weill F.X. 2014. Supplement 2008-2010 (no. 48) to the White-Kauffmann-Le Minor scheme. Res. Microbiol. 165(7):526-530. <http://dx.doi.org/10.1016/j.resmic.2014.07.004> <PMid:25049166>

Jackson B.R., Griffin P.M., Cole D., Walsh K.A. \& Chai S.J. 2013. Outbreakassociated Salmonella enterica serotypes and food commodities, United States, 1998-2008. Emerg. Infect. Dis. 19(8):1239-1244. <http://dx.doi. org/10.3201/eid1908.121511> <PMid:23876503>

Kay R.S., Vandevelde A.G., Fiorella P.D., Crouse R., Blackmore C., Sanderson R., Bailey C.L. \& Sands M.L. 2007. Outbreak of healthcare-associated infection and colonization with multidrug-resistant Salmonella enterica serovar Senftenberg in Florida. Infect. Control Hosp. Epidemiol. 28(7):805-811. <http://dx.doi.org/10.1086/518458> <PMid:17564982>

Marin C. \& Lainez M. 2009. Salmonella detection in feces during broiler rearing and after live transport to the slaughterhouse. Poult. Sci. 88(9):1999-2005. <http://dx.doi.org/10.3382/ps.2009-00040> <PMid:19687287>

Mattiello S.P., Drescher G., Barth Junior V.C., Ferreira C.A. \& Oliveira S.D. 2015. Characterization of antimicrobial resistance in Salmonella enterica strains isolated from Brazilian poultry production. Antonie Van Leeuwenhoek 108(5):1227-1238. <http://dx.doi.org/10.1007/s10482-015-0577-1> <PMid:26337044>

Medeiros M.A.N., Oliveira D.C.N.D., Rodrigues D.D.P. \& Freitas D.R.C.D. 2011. Prevalence and antimicrobial resistance of Salmonella in chicken carcasses at retail in 15 Brazilian cities. Revta Panam. Salud Publica 30(6):555-560. <http://dx.doi.org/10.1590/S1020-49892011001200010><PMid:22358402>

Mezalira T.S., Otutumi L.K., Moura R.A., Meza S.K.L., Goncalves D.D., Lima E.T., Messa V., Vieira B.G. \& De Brito D.C. 2014. Assessment of Salmonella spp. presence among broilers of naked neck Label Rouge lineage in Northwest region of Paraná State, Brazil. Afr. J. Microbiol. Res. 8(38):3471-3474. <http://dx.doi.org/10.5897/AJMR2014.6833>

Moreira G.D.N., Rezende C.S.M., Carvalho R.N., Mesquita S.Q.P.D., Oliveira A.N.D. \& Arruda M.L.T. 2008. Ocorrência de Salmonella spp. em carcaças de frangos abatidos e comercializados em municípios do estado de Goiás. Revta Inst. Adolfo Lutz 67(2):126-130. 
OIE 2015. List of antimicrobial agentes of veterinary importance. Disponível em <http://www.oie.int/fileadmin/Home/eng/Our_scientific_expertise/docs/ pdf/Eng_OIE_List_antimicrobials_May2015.pdf> Acesso em 10 dez. 2016.

Oliveira W.F., Cardoso W.M., Salles R.P.R., Romão J.M., Teixeira R.S.C., Câmara S.R., Siqueira A.A. \& Marques L.C.L. 2006. Initial identification and sensitivity to antimicrobial agents of Salmonella spp. isolated from poultry products in the state of Ceará, Brazil. Revta Bras. Ciênc. Avícolas 8(3):193-199.

Palermo-Neto J. \& Borsoi A. 2013. Antibioticoterapia em avicultura. Avicultura Industrial 8:37-44.

Pandini J.A., Pinto F.G.S., Muller J.M., Weber L.D. \& Moura A.C. 2015. Ocorrência e perfil de resistência antimicrobiana de sorotipos de Salmonella spp. isolados de aviários do Paraná, Brasil. Arqs Inst. Biológico, 82:1-6.

Panzenhagen P.H.N., Aguiar W.S., Frasão B.S., Pereira V.L.A., Abreu D.L.C., Rodrigues D.P., Nascimento E.R. \& Aquino M.H.C. 2016. Prevalence and fluoroquinolone resistance of Campylobacter and Salmonella isolates from poultry carcasses in Rio de Janeiro, Brazil. Food Control 61:243-247. <http://dx.doi.org/10.1016/j.foodcont.2015.10.002>

Ravagnani L.K., Agostinis R.O., Otutumi L.K., Lima E.T., Fernande J.I.M. \& Martins L.A. 2012. Pesquisa de Salmonella spp. em frangos de corte criados em galpões climatizados de uma integração na região Oeste do Paraná. Semina, Ciênc. Agrárias 33(6):2327-2336.

Rushdy A.A., Stuart J.M., Ward L.R., Bruce J., Threlfall E.J., Punia P. \& Bailey J.R. 1998. National outbreak of Salmonella Senftenberg associated with infant food. Epidemiol. Infect. 120(2):125-128. <http://dx.doi.org/10.1017/ S0950268897008546><PMid:9593480>

Shah D.H., Paul N.C., Sischo W.C., Crespo R. \& Guard J. 2017. Population dynamics and antimicrobial resistance of the most prevalent poultry-associated Salmonella serotypes. Poult. Sci. 96(3):687-702. <PMid:27665007>

Shirota K., Umali D.V., Suzuki T. \& Katoh H. 2012. Epizzootiologic role of feeds in the epidemiology of Salmonella Senftenberg contamination in commercial layer farms in eastern Japan. Avian Dis. 56(3):516-520. <http://dx.doi. org/10.1637/9964-101611-Reg.1> <PMid:23050468>

USDA 1998. Isolation and identification of Salmonella from meat, poultry, pasteurized egg, and siluriformes (fish) products and carcass and environmental sponges, method number 4.09. Food Safety and Inspection Service, Microbiology Laboratory Guide Book. 3rd ed. Disponível em <https://www.fsis.usda.gov/wps/portal/fsis/topics/science/laboratoriesand procedures/guidebooks-and-methods/microbiology-laboratoryguidebook> Acesso em 15 jan. 2016.
UBABEF 2011. A saga da avicultura brasileira: como o Brasil se tornou o maior exportador mundial de carne de frango. Coordenação Sergio Costa, tradução Vice Versa Tradução Escrita e Interpretação. Insight, Rio de Janeiro; UBABEF, São Paulo. 120p.

Van Boeckel T.P., Brower C., Gilbert M., Grenfell B.T., Levin S.A., Robinson T.P., Teillant A. \& Laxminarayan R. 2015. Global trends in antimicrobial use in food animals. Proc. Natl Acad. Sci. 112(18):5649-5654. <http://dx.doi. org/10.1073/pnas.1503141112><PMid:25792457>

Van Doren J.M., Neil K.P., Parish M., Gieraltowski L., Gould L.H. \& Gombas K.L. 2013. Foodborne illness outbreaks from microbial contaminants in spices, 1973-2010. Food Microbiol. 36(2):456-464. <http://dx.doi.org/10.1016/j. fm.2013.04.014><PMid:24010629>

Van Hoorebeke S., Van Immerseel F., De Vylder J., Ducatelle R., Haesebrouck F, Pasmans F., Kruif A. \& Dewulf J. 2009. Faecal sampling underestimates the actual prevalence of salmonella in laying hen flocks. Zoonoses Publ. Health 56(8):471-476. <http://dx.doi.org/10.1111/j.1863-2378.2008.01211.x> $<$ PMid:19243563>

Voss-Rech D., Vaz C.S.L., Alves L., Coldebella A., Leão J.A., Rodrigues D.P. \& Back A. 2015. A temporal study of Salmonella enterica serotypes from broiler farms in Brazil. Poult. Sci. 94(3):433-441. <http://dx.doi.org/10.3382/ ps/peu081><PMid:25595481>

WHO 2016a. Salmonella (non-typhoidal). Factsheet 139. Disponível em <http://www.who.int/mediacentre/factsheets/fs139/en/> Acesso em 10 dez. 2016

WHO 2016b. Critically important antimicrobials for human medicine. Ranking of Antimicrobials Agents for Risk Management of Antimicrobial Resistance due to Non-human Use. 5th Revision. Disponível em <http:// www.who.int/foodsafety/publications/antimicrobials-fifth/en/> Acesso em 29 jun. 2017.

Yamatogi R.S., Oliveira H.C., Possebon F.S., Pantoja J.C.F., Joaquim J.G.F., Pinto J.P.A.N. \& Araújo Junior J.P. 2016. Qualitative and quantitative determination and resistance patterns of Salmonella from poultry carcass. J. Food Protect. 79(6):950-955. <http://dx.doi.org/10.4315/0362-028X.JFP-15-489> <PMid:27296598>

Zweifel C. \& Stephan R. 2012. Spices and herbs as source of Salmonellarelated foodborne diseases. Food Res. Int. 45(2):765-769. <http://dx.doi. org/10.1016/j.foodres.2011.02.024> 